C-A/AP/\#145

March 2004

\title{
Transverse coupling impedance of the AGS A5 Injection Kicker Magnet
}

\author{
H. Hahn and W. Zhang
}

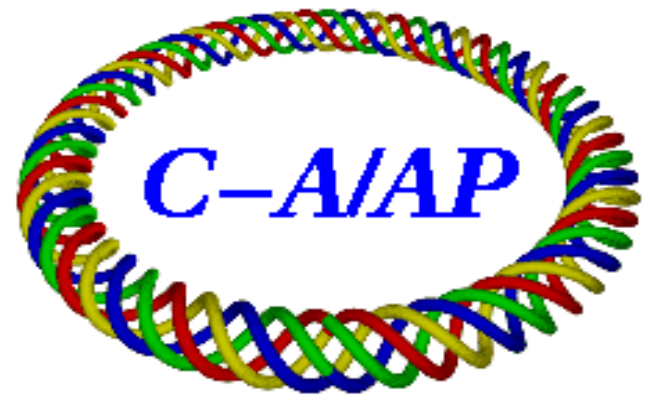

Collider-Accelerator Department Brookhaven National Laboratory

Upton, NY 11973 


\title{
Transverse coupling impedance of the AGS A5 Injection Kicker Magnet System
}

\author{
H. Hahn and W. Zhang
}

\section{Introduction}

The quest for higher intensities in accelerators/colliders depends to a large degree on the success in minimizing coupling impedances. Several projects under discussion at BNL are based on increased AGS intensities so that impedance reduction has become a current topic. Transverse instabilities are observed in the AGS at transition [1], with the A5 injection kicker magnet suspected to be a possible impedance contributor. The availability of a spare A5 kicker and equipment developed for the SNS extraction kicker allowed transverse impedance measurements without great effort [2]. This note presents the results for the transverse coupling impedances, both in horizontal and vertical direction, of the A5 kicker magnet system.

\section{The Magnet}

The A5 injection fast kicker consists of three full aperture picture frame lumped magnets [3]. The major dimensions of the 10 in. long magnet are given in Fig. 1 [4]. The magnets are spaced apart by 2.5 inches. The estimated inductance of the single magnets is found from $L=\mu_{0} \frac{w \ell}{g} \approx 0.74 \mu H$.

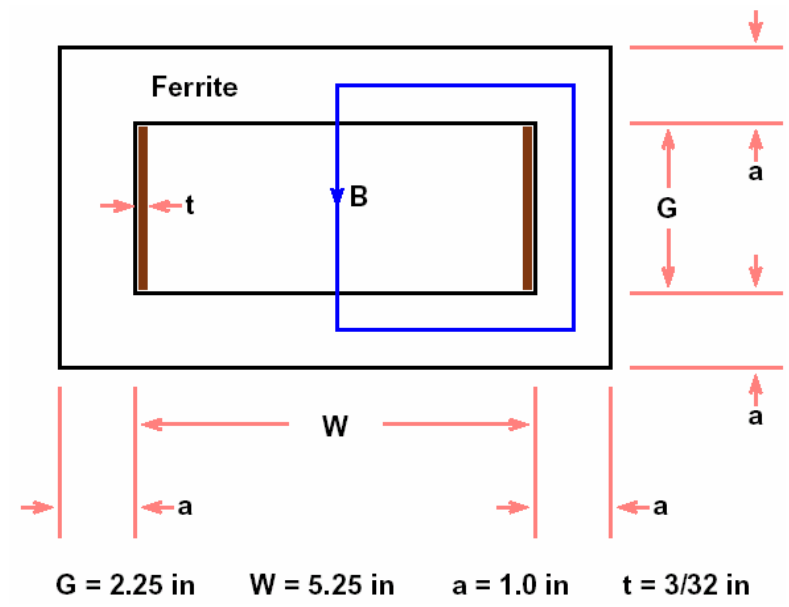

FIG. 1. Magnet Cross Section (courtesy ref. 4)

The input impedance of the three magnets, measured at the individual feedthrough terminals, is shown in Fig. 2. The curve for the center magnet is shown with the other two open (CTR) or shorted CTR (shrt). At this scale, the impedance is independent of the state, i.e. open or shorted, of the other two magnets: The magnets are magnetically uncoupled. The measured low frequency inductance, taken at $f=1.5915 \mathrm{MHz}$, is $L \approx 1.02 \mu H$, a value higher than the calculated due to end effects and the contribution 
from the feed through.. Note the resonance at $\sim 26.5 \mathrm{MHz}$, which involves a capacitance of $35 \mathrm{pF}$, which comes from the feed through and other stray values.
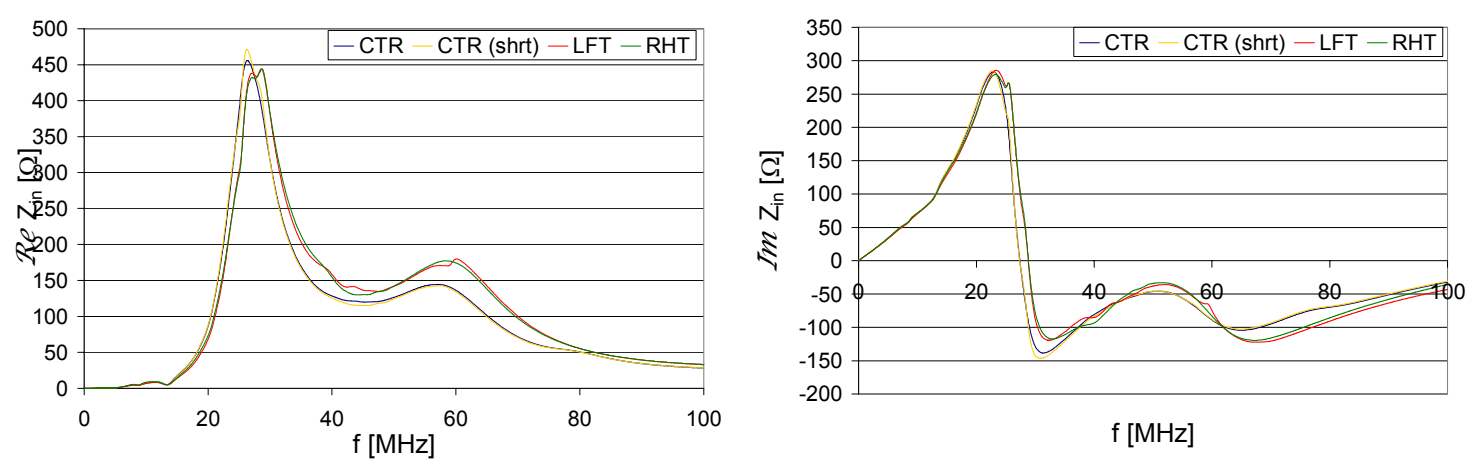

FIG. 2. Input impedance of magnets at feed through

\section{Twin-Wire Measurements}

The transverse coupling impedance of a kicker magnet can be measured on the bench by using the standard method in which a twin-wire (TW) "Lecher" line, simulating the beam, is inserted into the "Device Under Test (DUT)". Using a network analyzer, the forward transmission coefficients $S_{21}^{D U T}$ of the kicker is determined and compared with the $S_{21}^{R E F}$ obtained in a reference measurement. The use of a beam tube with the accelerator dimensions is appropriate. Here, a round tube with $14.6 \mathrm{~cm}$ (5.75 in.) inner diameter was taken.

The scattering coefficients were measured with the network analyzer, Agilent $8753 \mathrm{ES}$, set for a linear frequency range from $30 \mathrm{kHz}$ to $100 \mathrm{MHz}$, with 1601 points. The low-frequency instrument errors of noise and drift were reduced by using a $100 \mathrm{~Hz}$ IF-bandwidth, $0.1 \%$ smoothing, and by averaging over several sweeps. All results were obtained as average of three sweeps. The scattering coefficients are interpreted according to the standard HP-formula for lumped elements [5],

$$
Z^{D U T}=2 R_{C}\left(S_{21}^{R E F} / S_{21}^{D U T}-1\right),
$$

with $R_{C}$ the nominal instrument impedance, a value defined by the measurement setup to approximate the TW line impedance.

The transverse coupling impedance now follows from the standard twin-wire measurement expression, as

$$
Z_{\perp}=\frac{c}{\omega \Delta^{2}} Z^{D U T}
$$

The transverse impedance has values in vertical and horizontal direction. The measurements in horizontal, that is the kick direction, are here identified by $Z_{x}$ rather than the generic $Z_{\perp}$. 
For distributed impedances, the scattering coefficient is interpreted by the logformula,

$$
Z^{D U T}=-2 R_{C} \ln \frac{S_{21}^{D U T}}{S_{21}^{R E F}}
$$

It is justified to consider the individual magnet as a lumped element. However, the kicker with all magnets shorted and the vertical impedance intuitively appear as distributed devices. The $S_{21}$-ratios for these cases were interpreted either way but any differences are insignificant. Thus, the final results presented in this paper were obtained by using the conversion in the network analyzer corresponding to the standard lumped formula.

The impedance measurements were performed by using a twin-wire line, $126 \mathrm{~cm}$ long, home-made from $5 \times 7.5 \mathrm{~mm}$ rectangular tubes as shown in Fig. 3. The wire spacing is adjustable. Clearly, the signal increases with the wire spacing and the signal to noise ratio is reduced, yet it is recommended to keep the wire spacing smaller than the half height of the magnet aperture in order to remain in the perturbation regime, here $d \ll 6 \mathrm{~cm}$ (horizontal) and $3 \mathrm{~cm}$ (vertical). The transverse impedance value depends quadratically on the effective wire spacing, $\Delta$. Applying the theoretical results for round to rectangular wires, one finds the effective spacing to be

$$
\Delta=d \sqrt{1-(b / d)^{2}} \text {. }
$$

For the twin-wire used, the effective spacing is given by the center-to-center distance of the wires, $\Delta \approx d$ or $40.6 \mathrm{~mm}$ for horizontal and $27.9 \mathrm{~mm}$ for vertical measurements.

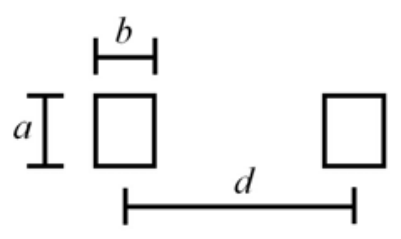

FIG. 3: The homemade twin-wire (TW) line. The dimensions are $a=7.5 \mathrm{~mm}, b=5$ $\mathrm{mm}$, and $d=40.6(\mathrm{H})$ or $27.9(\mathrm{~V}) \mathrm{mm}$.

Interpretation of the measurements requires the knowledge of the line characteristic impedance of the TW-line. The characteristic impedance was estimated to be $Z_{L} \approx 250 \Omega(\mathrm{H})$ from the theoretical expression for round wires by accommodating the rectangular lines with the use of $b^{\prime} \approx 2(a+b) / \pi$ instead of $\mathrm{b}$ in

$$
Z_{L}=\frac{c \mu_{0}}{\pi} \operatorname{acosh}\left(d / b^{\prime}\right)
$$

Although the bare TW-line can be used at the low frequency end, $<10 \mathrm{MHz}$, to determine the basic kicker magnet properties, it is for most coupling impedance measurements necessary to match the characteristic impedance to the $50 \Omega$ instrument impedance value. This can be done by resistive impedance matching or by the use of a transformer, or by a combination thereof. The nominal impedance $R_{C}$ is then given by the secondary side of the transformer with the resistors which provide the matching to the 
TW line. The characteristic line impedance is only approximately known, but mismatch errors are minimized and vanish at the low frequency end by the use of the nominal $R_{C}$.

A commercial (North Hills) wide-band transformers (NH15887, 50:200 $\Omega$, with $100 \mathrm{kHz}-100 \mathrm{MHz}$ ) with a center-tapped secondary winding, serving as $180^{\circ}$ hybrid, was used for the measurements. The reference forward scattering coefficient, $S_{21}^{R E F}$ of the assembled TW line in the beam tube is shown in Fig. 4.
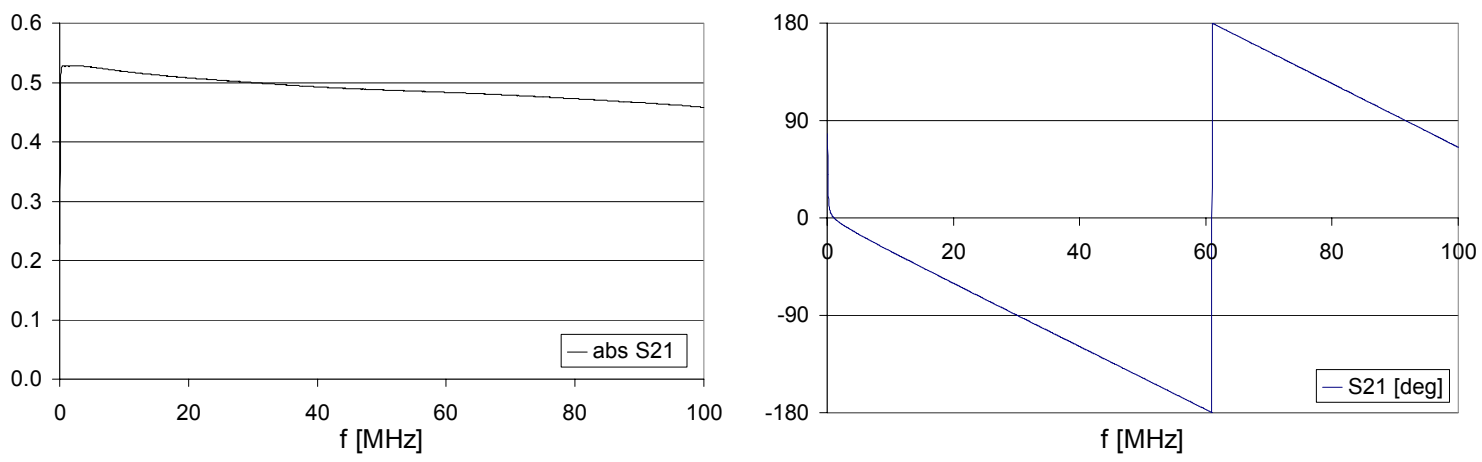

FIG. 4. Reference scattering coefficient

Matching is established by measuring the input impedance of the input transformer when the output transformer is terminated with the instrument impedance. The objective is a frequency-independent input impedance. The input impedance of the twin lines placed between the " $200 \Omega$ " transformers is shown in Fig. 5. A flat response was achieved by matching the lines with the transformer, $R_{0}=200 \Omega$ to $R_{C} \approx 250 \Omega$, the value to be used in the interpretative formulas. Resistive matching is done on the input side with a parallel resistor, $R_{P}$, and a series resistor, $R_{i n}$,

$$
\begin{aligned}
& R_{P}=R_{0} \sqrt{R_{C} /\left(R_{C}-R_{0}\right)} \approx 447 \Omega, \\
& R_{\text {in }}=R_{C}-R_{0} R_{P} /\left(R_{0}+R_{P}\right)=2 \times 56 \Omega,
\end{aligned}
$$

and a series resistor on the output side, $R_{\text {out }}=R_{C}-R_{0}=2 \times 27 \Omega$.

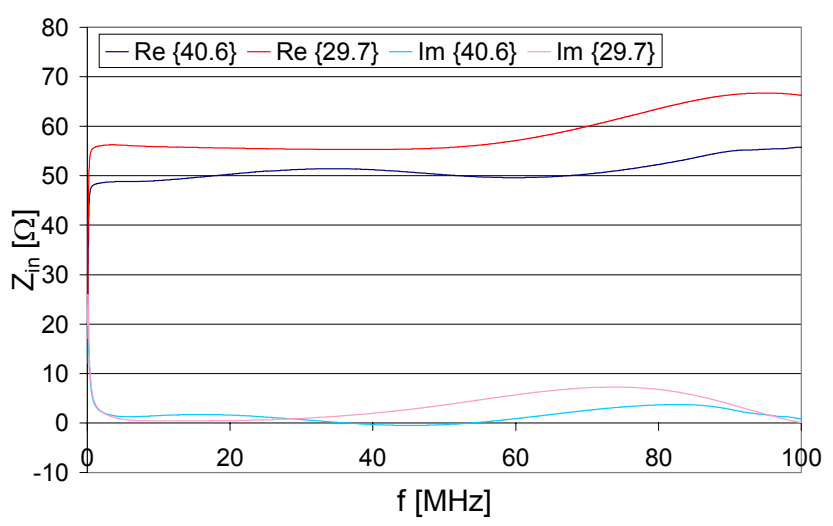

FIG. 5. Input impedance at transformer of TW line in tube. 


\section{Measured Device-under-Test impedances}

The setup for a twin wire measurement of a lumped kicker magnet, such as one of the three A5 injection kicker magnets is adequately represented by the equivalent circuit in Fig. 6. The TW line has an inductance, $L_{C}$ and a characteristic impedance, $Z_{L} \approx R_{C}$. There is a small difference in inductance and impedance between the line in the reference tube or in the magnet, but here it can be neglected. The total impedance of a lumped kicker magnet is composed of the coupled part, which reflects the external termination, $Z_{g}$ plus an uncoupled part due to image currents, represented by, $L_{I}$. The image current is primarily inductive but at higher frequencies can have a resistive part due to the ferrite material. The "stray" inductance leads to a difference between the impedance seen by the beam and measured by the TW method. In a typical case with the wire spacing small compared to the magnet aperture, this difference is inductive and can be neglected. Furthermore, $n=\Delta / g$ and $\kappa \ll 1$.

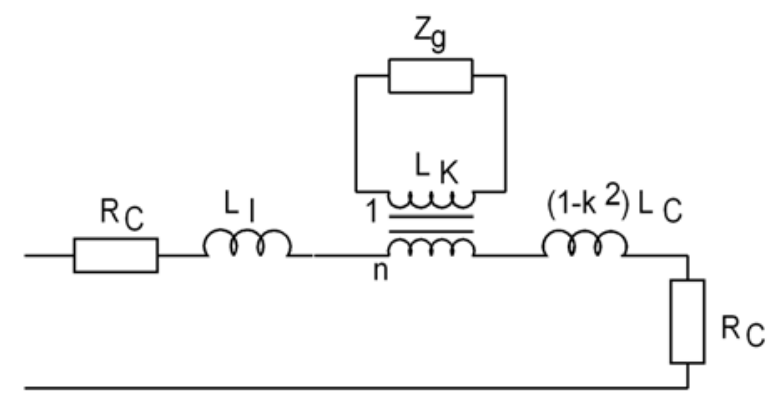

FIG. 6. Lumped kicker magnet circuit model

The equivalent circuit for the A5 kicker assembly has three individual transformers plus one image current impedance. The total impedance can be measured on the assembly as a single unit, each magnet terminated with $Z_{g}=12 \Omega+700 \mathrm{pF}$ in series. However the special configuration of this kicker is an opportunity to study the possibility of adding the individual contributions. The image impedance in horizontal direction is obtained by shorting the three magnet terminals. The vertical impedance is obtained from a single measurement.

As discussed above, the three magnets are not coupled and act as lumped elements. Interpretation of the $S_{21}$ ratio via the HP-formula seems appropriate. However, the image impedance seen with the magnet terminals shorted, could be seen as a distributed impedance. As verification, the $S_{21}$-ratios for these cases were interpreted via the $\log$ and HP formula, but any differences are insignificant. The measured $S_{21}$ ratio of the kicker with the three magnets terminated (3RC)/open(OPN)/shorted(SHRT) at their feedthrough are evaluated via the alternate formulas and the resulting $Z^{D U T}$ is shown in Fig. 7. 

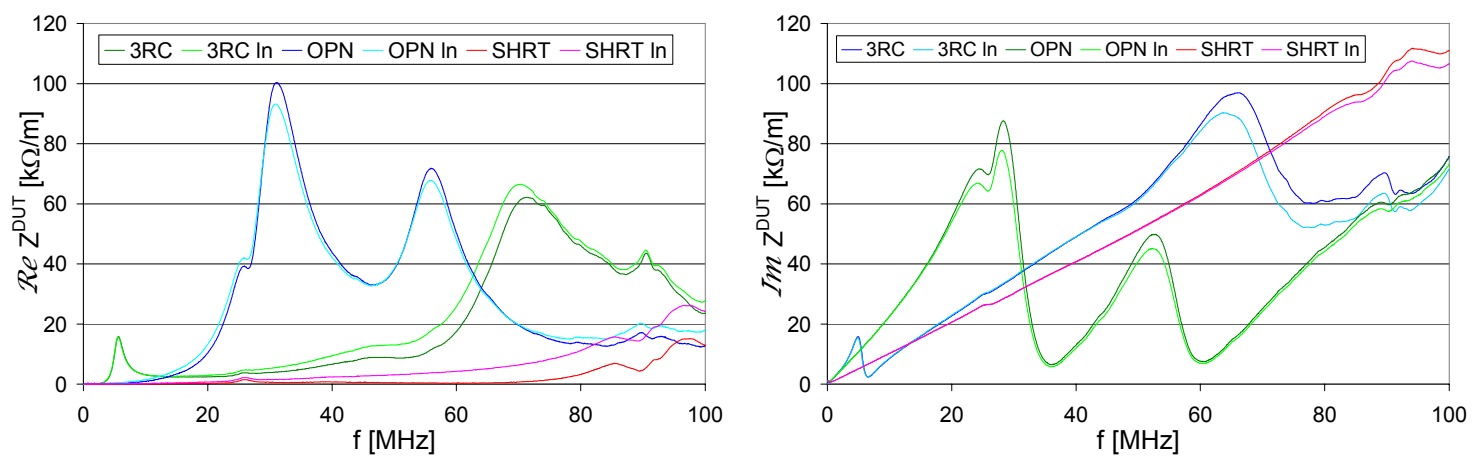

FIG. 7. Measured $Z^{D U T}$ via HP and $\log$ formulas.

The impedance of each magnet terminated by the simulated value of $12 \Omega$ in series with $700 \mathrm{pF}$ (with the other two shorted ) is shown in Fig. 8. The sum of the three magnets, nota bene after subtraction of the two excess image impedance values, is compared to the value obtained from the single measurement with all three terminations terminated with the RC. The agreement is satisfactory and confirms the independence of the three magnets. The total result, identified in the figure as $3 \mathrm{RC}$, is used to calculate the horizontal coupling impedance.
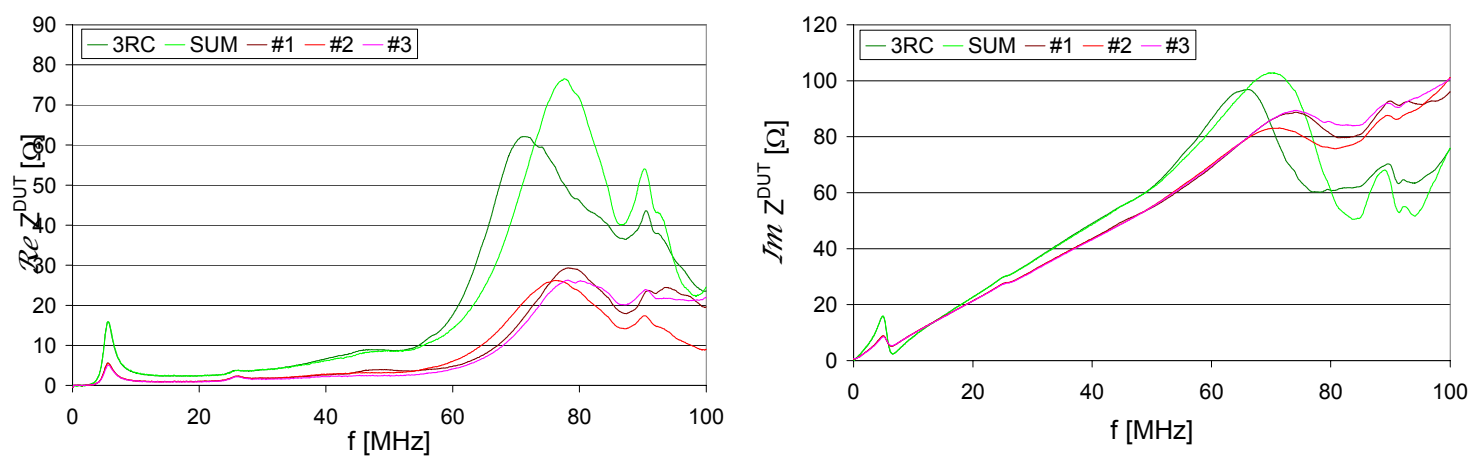

FIG. 8. DUT impedance of kicker, total (green) and sum of three magnets.

The vertical impedance is measured with the narrow Twin-Wire line ( $\Delta=27.9$ $\mathrm{mm}$ ) to remain below the magnet gap of $57.2 \mathrm{~mm}$. The DUT impedance for the kicker with all magnets shorted or open is shown in Fig. 9. Again, the $S_{21}$ data is interpreted both according to the HP as well as the log formulas, but here the vertical transverse coupling impedance has been calculated from the red log values. Note that the state of the magnet terminals, shorted or open, has minimal effect on the vertical impedance. Thus, the resonance at $\sim 40 \mathrm{MHz}$ is not generated by the load, however its source has not been determined. 

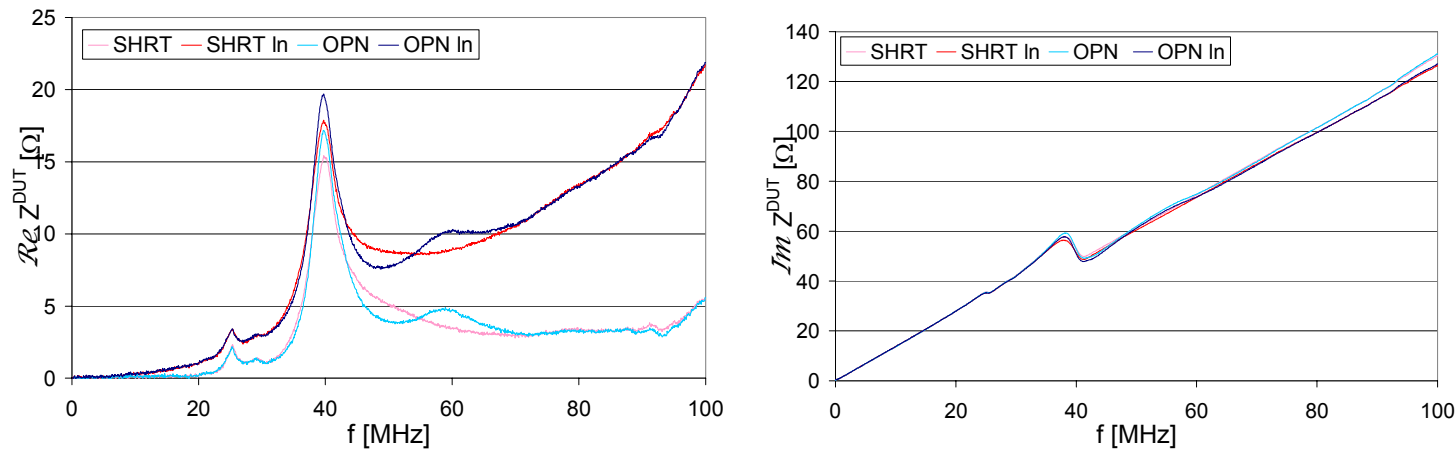

FIG. 9. Vertical DUT impedance of the kicker

\section{Transverse coupling impedance}

The transverse impedance, both horizontal (H) and vertical (V), is shown in Fig. 10.
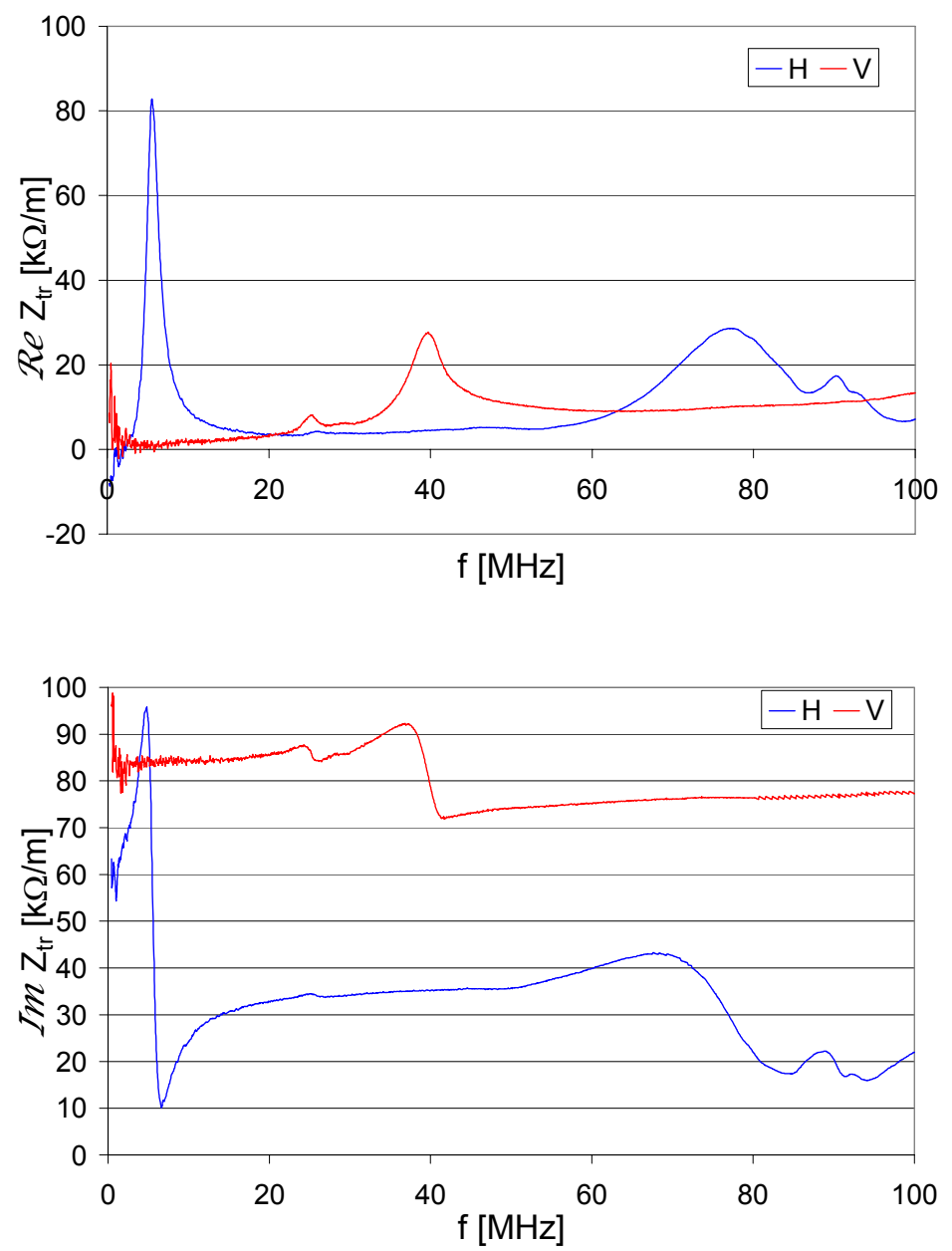

FIG. 10. Transverse coupling impedances of the AGS A5 injection kicker 


\section{Acknowledgements.}

The present study was initiated at the suggestion of W. Glenn. The technical help from members of the Beam Components group and of the Pulsed Power group is gratefully acknowledged.

\section{References}

1. W. Glenn, private communication (2004)

2. H. Hahn, BNL/SNS Technical Note No 120, (2003)

3. W. Zhang, R. Sanders, A. Soukas, and J. Tuozzolo, Proc. PAC, New York, NY 1999, p. 1264

4. L.A. Ahrens and C. J. Gardener, Report C-A/AP/91 (2002)

5. H. Hahn, Phys. Rev. ST Acc. Beams, 7, 012001 (2004) 